\title{
High-flow Nasal Cannula: Mechanisms of Action and Adult and Pediatric Indications
}

\author{
Frank J. Lodeserto ${ }^{1}$, Thomas M. Lettich ${ }^{1}$, Salim R. Rezaie ${ }^{2}$ \\ 1. Internal Medicine, Geisinger Medical Center, Danville, USA 2. Emergency Medicine, Methodist Hospital, San \\ Antonio, USA
}

Corresponding author: Frank J. Lodeserto, frank.lodeserto@gmail.com

\begin{abstract}
The use of the heated and humidified high-flow nasal cannula has become increasingly popular in the treatment of patients with respiratory failure through all age groups. This article will examine the main mechanisms of actions attributed to the use of the high-flow nasal cannula and review the indications in adult and pediatric populations (outside of the neonatal period). It is unclear which of the mechanisms of action is the most important, but it may depend on the cause of the patient's respiratory failure. This article describes the mechanism of action in an easy to remember mnemonic (HIFLOW); Heated and humidified, meets Inspiratory demands, increases Functional residual capacity (FRC), Lighter, minimizes Oxygen dilution, and Washout of pharyngeal dead space. We will also examine some of the main indications for its use in both the adult and pediatric age groups. The data for the use of high-flow nasal cannula is growing, and currently, some of the main adult indications include hypoxemic respiratory failure due to pneumonia, post-extubation, pre-oxygenation prior to intubation, acute pulmonary edema, and use in patients who are "do not resuscitate or intubate". The main pediatric indication is in infants with bronchiolitis, but other indications are being studied, such as its use in asthma, croup, pneumonia, transport of a critically ill child, and post-extubation.
\end{abstract}

Received 10/29/2018

Review began 11/03/2018 Review ended 11/14/2018 Published 11/26/2018

๑) Copyright 2018

Lodeserto et al. This is an open access article distributed under the terms of the Creative Commons Attribution License CC-BY 3.0., which permits unrestricted use, distribution, and reproduction in any medium, provided the original author and source are credited.
Categories: Emergency Medicine, Internal Medicine, Pediatrics

Keywords: respiratory failure, dead space washout, oxygen dilution, high-flow nasal cannula

\section{Introduction And Background}

The heat and humidified high-flow nasal cannula or, as most call it, high-flow nasal cannula (HFNC), isn't just a standard nasal cannula turned up to very high flow rates. It takes gas, is able to heat it to $37^{\circ} \mathrm{C}$ with a $100 \%$ relative humidity, and can deliver $0.21-1.00 \%$ fraction of inspired oxygen $\left(\mathrm{FiO}_{2}\right)$ at flow rates of up to 60 liters $(\mathrm{L}) / \mathrm{min}$. The flow rate and $\mathrm{FiO}_{2}$ can be independently titrated based on a patient's flow and $\mathrm{FiO}_{2}$ requirements.

There are two main companies who manufacture these devices: Vapotherm ${ }^{\circledR}$ (Exeter, NH), which has a device that can deliver flow rates of up to $50 \mathrm{~L} / \mathrm{min}$ flow rates, and Fisher and Paykel Healthcare, Inc. (Auckland, New Zealand), who offers both the Optiflow ${ }^{\mathrm{TM}}$, as well as the AIRVO ${ }^{\mathrm{TM}} 2$ device, both of which can deliver flow rates of up to $60 \mathrm{~L} / \mathrm{min}$.

Each company offers smaller cannula sizes for premature neonates, as well as children of various ages, to adult-sized cannulas. The nasal prongs should fit snuggly in the patient's nares in order to prevent entrainment of room air around the cannula, a problem which occurs in standard nasal cannulas. Each manufacturer has a maximum flow rate for each cannula size corresponding to the size and age of the patient.

\section{Review}

\section{Mechanisms of actions}

There are many beneficial mechanisms of action that have been attributed to the effectiveness of high-flow nasal cannula in adult and pediatric patients with respiratory failure. It is not clear which of the benefits are most important, and it may depend on the individual patient's etiology of respiratory failure. The mechanisms of action below are not listed in the order of importance but rather to give the reader an easy to remember mnemonic (HIFLOW) for the mechanisms that have been attributed to the use of high-flow nasal oxygen.

Heated and Humidified

Heated and humidified oxygen has a number of benefits compared to standard oxygen therapy. Standard oxygen therapy delivered through a nasal cannula or another device, such as a non-rebreather mask 
(NRBM), delivers cold (not warmed) and dry (not humidified) gas. This cold, dry gas can lead to airway inflammation, increase airway resistance, and impair mucociliary function, possibly impairing secretion clearance [1]. Also, a significant amount of energy is expended by individuals to both warm and humidify gas during normal breathing [2]. Thus, heated and humidified oxygen may improve secretion clearance, decrease airway inflammation, and also decrease energy expenditure, particularly in the setting of acute respiratory failure [1-2].

Inspiratory Demands

One obvious benefit is that the high-flow nasal cannula can deliver very high flow rates of gas in an attempt to match a patient's inspiratory flow demands. This is important as patients in acute respiratory failure can become extremely tachypneic, and their peak inspiratory flows (PIF), which may normally be $30 \mathrm{~L} / \mathrm{min}-60$ $\mathrm{L} / \mathrm{min}$ at rest, can reach upwards of $120 \mathrm{~L} / \mathrm{min}$ in acute respiratory failure [3]. If these patients with respiratory failure (with PIF rates of up to 60 - $120 \mathrm{~L} / \mathrm{min}$ and high minute volumes (> $20 \mathrm{~L} / \mathrm{min}$ in some adults)) are placed on a $15 \mathrm{~L} / \mathrm{min}$ NRB mask, then this may not provide adequate support. This will be discussed later in this review when we discuss the concept of oxygen dilution. One of the main mechanisms to improve a patient's work of breathing is to attempt to match their peak inspiratory flow demands with the use of a high-flow device.

Functional Residual Capacity

There is some debate over the level of positive end-expiratory pressure (PEEP) provided by high-flow devices. Best estimates are $1 \mathrm{~cm} \mathrm{H}_{2} 0$ of PEEP for every $10 \mathrm{~L} /$ min of flow delivered with closed mouth breathing [4-5].

There has been a lot of variation in studies measuring how much PEEP that high-flow cannulas can generate. This may vary from patient to patient as there are many factors that can affect how much PEEP can actually be delivered to a patient. Factors, such as the patient's size (obese, adult, child), the liter flow rate being delivered (L/min), and mouth open versus mouth closed breathing (pressure may escape when a patient's mouth is open), can all affect the amount of PEEP being delivered [4].

The debate can continue, but it appears that HFNC can increase a patient's functional residual capacity (FRC) or the lung volume at the end of expiration, which is something that PEEP usually improves. A study by Riera et al. showed the use of HFNC increased end-expiratory lung impedance (EELI), implying there was an improvement in FRC [6]. They used electrical impedance tomography (EIT), a noninvasive, real-time imaging method that provides a cross-sectional ventilation image of the lung, to demonstrate an increased EELI.

It also appears that the use of HFNC can decrease preload by increasing intrathoracic pressure, again another feature commonly attributed to the addition of PEEP. Roca et al. demonstrated in a sequential interval study on 10 patients (New York Heart Association (NYHA) Classification III - heart failure but not in an acute congestive heart failure (CHF) exacerbation) that the use of HIFLOW caused an inspiratory collapse of the inferior vena cava (IVC) from the patient's baseline which was measured by echocardiogram [7].

High-flow nasal cannula use seems to cause alveolar recruitment and increased FRC, as well as increased intrathoracic pressure, likely as a result of the added PEEP; however, it is not certain if perhaps another mechanism may be responsible for these findings.

Lighter

Patients often prefer the use of HFNC to that of non-invasive continuous or bilevel positive pressure ventilation (CPAP or BPAP) because the tight-fitting mask can be uncomfortable for some patients. They may even prefer it to the standard nasal cannula (NC) because of the warmed, humidified gases that won't dry their mucosa like standard oxygen therapy [5]. This may lead to higher compliance with HFNC and perhaps an improvement in the patient's oxygenation and work of breathing.

$\mathrm{O}_{2}$ Dilution

It is taught that $1 \mathrm{~L} /$ min administered via nasal cannula will deliver $\sim 4 \% \mathrm{FiO}_{2}$ above room air (21\%). Thus, 1 $\mathrm{L} / \mathrm{min}$ via the $\mathrm{NC}$ should deliver $\sim 25 \% \mathrm{FiO}_{2}$, while $2 \mathrm{~L} / \mathrm{min}$ should deliver $29 \% \mathrm{FiO}_{2}$ (Table 1). Many refer to this as the " $1: 4$ rule", and this concept that is taught widely; let's examine this more closely. 


\section{Cureus}

Table 1

\begin{tabular}{l|l} 
Liter Flow & $\mathrm{FiO}_{2}$
\end{tabular}

$1 \quad 25 \%$

$2+29 \%$

$3 \quad 33 \%$

$\begin{array}{ll}4 & 37 \%\end{array}$

TABLE 1: "4:1" Rule

$\mathrm{FiO}_{2}$ : fraction of inspired oxygen

Consider a $70 \mathrm{~kg}$ male patient breathing 30 - 40 beats per minute (bpm) with normal tidal volumes ( $\sim 500$ $\mathrm{mL}$ 's) who develops acute hypoxemia. This patient's minute ventilation would be between $15-20 \mathrm{~L} / \mathrm{min}$. If this patient is placed on $6 \mathrm{~L} / \mathrm{min} \mathrm{NC}$, this should theoretically deliver an $\mathrm{FiO}_{2} \sim 45 \%(6 \mathrm{~L} \mathrm{x} 4 \%=24+$ room air $(21 \%)=45 \%$ ) if the "1:4 rule" holds true. If this patient is breathing $15-20 \mathrm{~L}$ through his mouth and nares (around the nasal cannula) at $21 \%$, then the gas reaching the patient's trachea will be diluted with room air and be closer to $21 \% \mathrm{FiO}_{2}$ rather than $45 \% \mathrm{FiO}_{2}$ (Figure 1).

\section{Oxygen Dilution}

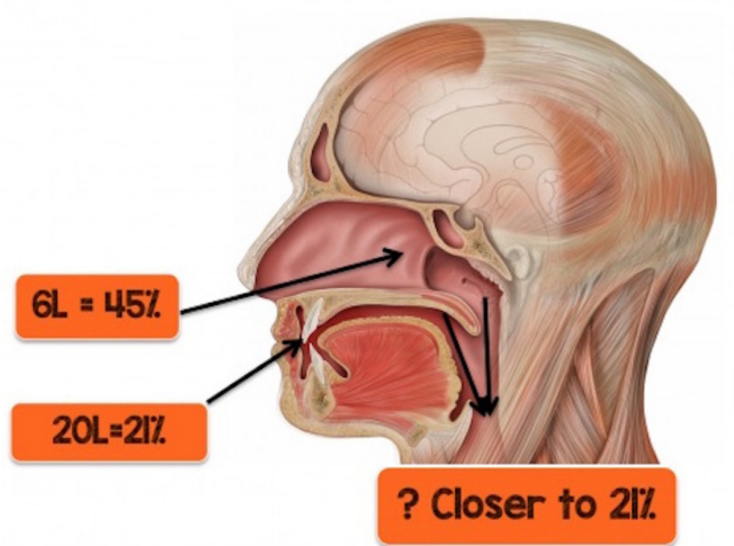

If there is a NC at 6 liter/min delivering $45 \%$., but your patient is breathing 20 liter/min at room air (2l\%). then what $\%$ fi02 do you think is actually reaching the patients trachea? I don't actually know but definitely NOT $45 \%$ and likely closer to $21 \%$. This phenomenon is known as oxygen dilution and will occur if you don't meet or exceed your patients inspiratory flow demands.

\section{FIGURE 1: Oxygen Dilution}

$\mathrm{FiO}_{2}$ : fraction of inspired oxygen; L: liter; NC: nasal cannula

Image courtesy of www.rebelem.com

To deliver higher amounts of $\mathrm{FiO}_{2}$ effectively to a patient, the patient's minute ventilation and inspiratory demands need to be not only matched but to exceed to minimize the effects of oxygen dilution (Figure 2). 


\section{Cureus}

\section{On HiFlow}

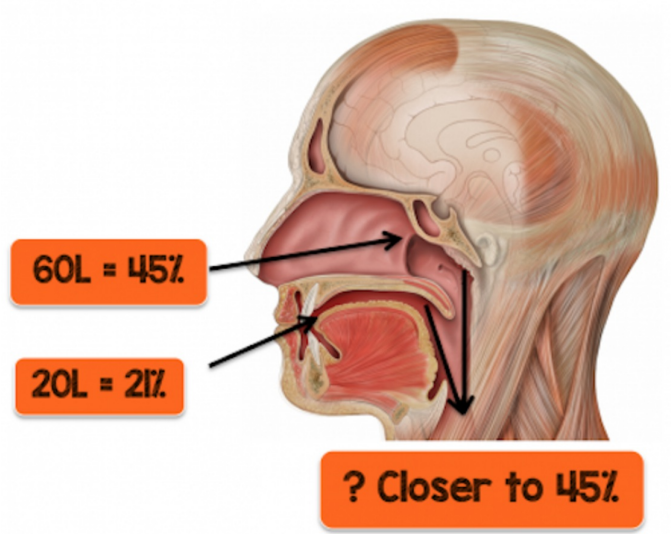

Now place your patient on a hiflow NC delivering $60 \mathrm{~L}$ at $45 \%$, with your patient still breathing 20 liter/min at room air (21\%), and what $\%$ fi02 do you think is actually reaching the patients trachea? I still don't actually know, but I believe it will now be closer to $45 \%$. To deliver higher fiO2 concentrations you must not only match, but exceed your patients inspiratory flow to minimize oxygen dilution.

\section{FIGURE 2: Minimizing Oxygen Dilution}

$\mathrm{FiO}_{2}$ : fraction of inspired oxygen; L: liter; NC: nasal cannula

Image courtesy www.rebelem.com

\section{Washout of Dead-space}

We can normally rebreathe a third of our previously expired tidal volume, and instead of breathing $21 \%$ (room air) and negligible amounts of carbon dioxide, we may rebreathe more like 15 - 16\% oxygen and 5 - $6 \%$ carbon dioxide. This is because the previously exhaled breath (low in oxygen and containing carbon dioxide) is not fully exhaled and remains in the upper airway. When the patient takes their next breath from atmospheric gas, not all of that gas will actually enter the alveoli. In fact, it's a mixture of the new atmospheric gas $\left(21 \% \mathrm{FiO}_{2}\right.$, negligible $\left.\mathrm{CO}_{2}\right)$ and their previously exhaled gas $(<21 \%$ oxygen with a larger amount of $\mathrm{CO}_{2}$ ) that enters the alveoli for gas exchange. In patients with acute respiratory failure, the percentage of gas we rebreathe gets larger, and as a result, we can rebreathe larger amounts of carbon dioxide as we inspire from a mixed reservoir from our upper airway.

One of the major benefits of HFNC (some argue it's actually the main benefit) is that it gives you a continuous flow of fresh gas at high-flow rates replacing or washing out the patient's pharyngeal dead-space (the old gas low in oxygen and high in $\mathrm{CO}_{2}$ ). Each breath that the patient now re-breathes with high-flow nasal cannula will have had its carbon dioxide washed out and replaced with oxygen-rich gas and thus improving breathing efficiency [8].

Figure 3 (below) gives you an easy to remember mnemonic to recall the mechanisms of action for the highflow nasal cannula. 


\section{Cureus}



FIGURE 3: Mechanisms of Action of Flow

FRC: functional residual capacity

Image courtesy of www.rebelem.com

The majority of the benefits of the high-flow nasal cannula, as we have discussed above, come as a result of the high flow rates that can be delivered (Figure 4). Delivering heated and humidified oxygen has significant benefits, but to optimize the effectiveness of high-flow nasal cannula for the patient, ensure that the inspiratory flow is optimized. As we will discuss in our next section, pediatric data (particularly in bronchiolitis) shows that flow rates of $2 \mathrm{~L} / \mathrm{kg} / \mathrm{min}$ are effective and well tolerated up to maximum flow rates of $60 \mathrm{~L} / \mathrm{kg} / \mathrm{min}$ in adults.

\section{H: Heated \&. Humidified - Provides heated and humidified gas I: Inspiratory Demands - Can better meet elevated peak inspiratory flow demands}

\section{F: Functional Residual Capacity = Increases FRC likely via delivery of PEEP}

\section{L: Lighter = More easily tolerable than CPAP or BIPAP \\ O: Oxygen Dilution - Can minimize oxygen dilution by meeting flow demands}

\section{W: Washout of dead space = Provides high flow rates leading} to wash out of pharyngeal dead space (CO2 removal)

\section{FIGURE 4: Mechanisms of Action of High-Flow Nasal Cannula}

BiPAP: bilevel positive airway pressure; $\mathrm{CO}_{2}$ : carbon dioxide; CPAP: continuous positive airway pressure; FRC: functional residual capacity; PEEP: positive end-expiratory pressure

Image courtesy of www.rebelem.com 


\section{Adult indications}

Acute Hypoxemic Respiratory Failure (Mainly from Community-acquired Pneumonia)

Community-acquired pneumonia would seem like an ideal indication for the use of the high-flow nasal cannula. The heated, humidified oxygen should allow improved mobilization of secretions, and its ability to minimize oxygen dilution, meet inspiratory demands, and improve end-expiratory lung volumes all seem ideal in patients with pneumonia. HFNC may allow patients to cough, mobilize secretions, and be suctioned, if necessary, all benefits that are often difficult to achieve when a patient is on noninvasive positive pressure ventilation (NIPPV). The only exception would be in patients with acute chronic obstructive pulmonary disease (COPD) exacerbations from pneumonia, as there is evidence demonstrating a decreased mortality and need for intubation with the use of NIPPV [9].

Frat et al. conducted a multi-centered, open-label, randomized control trial with 300 patients, mainly with community-acquired pneumonia (CAP), randomized to either oxygen (non-rebreather mask), HFNC (for two days), or NIPPV (for at least 8 hrs/day for two days) [10]. The primary outcome was intubation rates, and the data did not show a statistically significant difference between the groups. Though this study seemed like a negative trial for the use of a high-flow nasal cannula, a secondary outcome (90-day all-cause mortality) showed that the use of HFNC, even after adjusting for illness severity (Acute Physiology and Chronic Health Evaluation (APACHE) II and cardiac insufficiency), was improved with the use of HFNC compared to NIPPV and standard oxygen therapy. A post hoc analysis also showed a statistically significant reduction in the intubation rates in patients with severe respiratory failure $\left(\mathrm{PaO}_{2} / \mathrm{FiO}_{2} \leqslant 200\right)$ using HFNC. A meta-analysis by Ni et al. also demonstrated that the use of HFNC compared to NIPPV and conventional oxygen therapy (COT) was associated with a reduction in endotracheal intubation rates in acute respiratory failure [11].

A recent study by Azoulay et al. comparing the use of the high-flow nasal cannula to standard oxygen therapy in immunocompromised patients with acute hypoxemic respiratory failure failed to show a difference in 28-day mortality between the groups [12]. Despite improvements in oxygenation (higher $\mathrm{PaO}_{2} / \mathrm{FiO}_{2}$ ratio compared to standard oxygen therapy), high flow nasal cannula also failed to demonstrate a difference in rates of intubation, intensive care unit (ICU) length of stay, ICUacquired infections, hospital length of stay or patient comfort and dyspnea scores.

Post-extubation (Patients at Low Risk for Reintubation)

Another unique use for high-flow nasal cannula is in patients after extubation who are at low-risk for needing reintubation (Table 2). Hernandez et al. performed a randomized control trial in 527 patients at lowrisk for needing reintubation within 72 hours from extubation [13]. Patients were extubated and then randomized to either standard oxygen therapy, which would be considered the standard of care in this group of low-risk patients, or to HFNC. The group extubated to the high-flow nasal cannula had a statistically significant lower rate of reintubation (4.9\%) compared to standard oxygen therapy (12.2\%). It is unclear why this benefit was seen, but this is certainly a group where many intensivists would not probably attempt the use of high-flow nasal cannula as a therapy as it is usually reserved for more high-risk patients. 


\section{Cureus}

Table 2

Low Risk for Reintubation Characteristics

Age $<65$

CHF was not an indication for intubation

APACHE II $<12$ on the day of extubation

$\mathrm{BMI}<30$

No airway patency problems

Able to manage secretions

$<2$ co-morbidities

Ventilated $<7$ days

\section{TABLE 2: Low Risk for Reintubation Characteristics}

APACHE II: Acute Physiology and Chronic Health Evaluation II; BMI: body mass index; CHF: congestive heart failure;

Table adapted from Hernandez et al. [11]

Pre-oxygenation Prior to Intubation

Intubation of a critically ill patient is a high-risk procedure with high rates of complications, including hypoxemia, hypotension, and even cardiac arrest [14]. The high-flow nasal cannula device has an advantage compared to alternative methods, such as bag-mask ventilation (BMV) and NIPPV. The high-flow nasal cannula device can stay on the patient and provide continued oxygen therapy, as well as possibly provide positive pressure, even during the apneic period compared to BMV and NIPPV, which have to be removed during the intubation procedure. A high-flow nasal cannula may be as effective as NIPPV and superior to standard oxygen therapy for pre-oxygenation prior to intubation in critical patients [14-16].

Do Not Resuscitate (DNR)/Do Not Intubate (DNI) in Respiratory Distress

Peters et al. demonstrated that HFNC may be an effective therapy for patients who are DNI with acute hypoxemia and mild hypercapnia $\left(\mathrm{pCO}_{2}<65\right)$ [17]. This therapy was well tolerated and provided acceptable oxygenation without the need for an escalation to NIPPV in $82 \%$ of the subjects. This device provides both therapeutic and palliative benefits and may allow for patients to be treated outside of the ICU

Cardiogenic Pulmonary Edema

There isn't a lot of convincing data to recommend the use of HFNC in patients with cardiogenic pulmonary edema; however, as mentioned earlier, we do know that it can increase intrathoracic pressure and, therefore, is likely to decrease preload. Makdee et al. demonstrated that the high-flow nasal cannula improved the severity of dyspnea in patients with acute cardiogenic pulmonary edema in the emergency department compared to oxygen therapy (NC or NRBM) [18]. More data is needed to demonstrate its effectiveness in this patient population; however, it may be a reasonable therapy for those who are not able to tolerate NIPPV.

\section{Pediatric indications}

Bronchiolitis

The majority of pediatric data supporting the use of high-flow nasal cannula outside of neonatal use is in bronchiolitis. Pediatric patients with mild to severe bronchiolitis have the most evidence to support its use. Franklin et al. conducted a multi-centered, randomized controlled trial comparing the use of high-flow nasal cannula (dose $=2$ liters per kilogram/min) to standard oxygen in 1,472 infants ( $<12$ months) with moderate to severe bronchiolitis [19]. Their primary outcome was a failure of treatment requiring an escalation of care. Only $12 \%$ or $87 / 739$ infants in the high-flow nasal cannula group failed therapy, while $23 \%$ or $167 / 733$ children in the standard oxygen group required an escalation of care. Failure of therapy was defined by having three out of four clinical signs, including persistent tachycardia, tachypnea, and oxygen desaturation, as well as an elevated pediatric early warning score. Interestingly, $61 \%$ or 102/167 infants who failed standard oxygen therapy were successfully rescued with the high-flow nasal cannula. No differences 
were noted in secondary outcomes, including the length of hospital stay, duration of oxygen therapy, pediatric intensive care unit (PICU) admission, or intubation rates.

Other studies have shown the use of HFNC may decrease the need for intubation [20-21], prevent ICU admission [18], and may be as efficacious as NIPPV in the prevention of intubation [21].

Other Uses in Pediatrics

Outside of bronchiolitis, there is limited data to support the use of HFNC in pediatrics. There is some growing evidence for its use in other disease processes where it theoretically may be beneficial. There have been some small retrospective trials examining its benefit with asthma [22-23]. The heated and humidified oxygen may be beneficial to further prevent airway inflammation and bronchospasm. The high-flow rates used with HFNC may also meet the inspiratory demands of the patient, but it may not be as effective in the delivery of aerosolized bronchodilators to distal airways.

Other uses may include pneumonia; however, a lack of data exists here as well and its use would largely be extrapolated from adult use in community-acquired pneumonia. Other disease processes, such as croup, have been retrospectively examined showing that there may exist some benefit [22], as well as in patients in the post-extubation phase after withdrawal of invasive mechanical ventilation [23].

A promising use may be in the transport of critically ill children to larger pediatric hospitals. Newer highflow devices use battery power and can now be portable to transport children on high-flow devices rather than having to intubate or use NIPPV. Its use appears to be as safe as NIPPV to transport critically ill children between hospitals [24].

\section{Conclusions}

Most of the benefits of the high-flow nasal cannula are from the high-flow rates it can deliver, as well as the heating and humidification of gas. For patients in acute respiratory distress, optimizing your flow rates early will ensure the benefits of the device. Flow rates of 2 liters per $/ \mathrm{kg} / \mathrm{min}$ appear to be safe and effective from pediatric trials up to maximum flow rates of 60 liters/min in adults.

The majority of the benefits, besides heating and humidification, come from the optimal flow. The mnemonic (HIFLOW) will help one to remember the mechanisms of action, including Heated and humidified, meets Inspiratory demands, increases Functional residual capacity (FRC), Lighter, minimizes Oxygen dilution, and Washout of pharyngeal dead space. The most important mechanism may depend on the individual patient's need for the high-flow nasal cannula.

The indications for the use of HFNC in adults include community-acquired pneumonia, post-extubation (even in low-risk patients), pre-oxygenation prior to intubation, DNI patients with respiratory failure, and perhaps in patients with cardiogenic pulmonary edema when NIPPV is not tolerated. In pediatric patients outside the neonatal period, the strongest evidence for the use of the high-flow nasal cannula exists in patients with bronchiolitis, but there is promising evidence that it may benefit other children; however, this data is still lacking.

\section{Additional Information \\ Disclosures}

Conflicts of interest: In compliance with the ICMJE uniform disclosure form, all authors declare the following: Payment/services info: All authors have declared that no financial support was received from any organization for the submitted work. Financial relationships: All authors have declared that they have no financial relationships at present or within the previous three years with any organizations that might have an interest in the submitted work. Other relationships: All authors have declared that there are no other relationships or activities that could appear to have influenced the submitted work.

\section{References}

1. Chidekel A, Zhu Y, Wang J, Mosko JJ, Rodriguez E, Shaffer TH: The effects of gas humidification with highflow nasal cannula on cultured human airway epithelial cells. Pulm Med. 2012, 2012:380686. $10.1155 / 2012 / 380686$

2. Dysart K, Miller TL, Wolfson MR, Shaffer TH: Research in high flow therapy: mechanisms of action . Respir Med. 2009, 103:1400-1405. 10.1016/j.rmed.2009.04.007

3. Katz JA, Marks JD: Inspiratory work with and without continuous positive airway pressure in patients with acute respiratory failure. Anesthesiology. 1985, 63:598-607.

4. Parke RL, McGuinness SP: Pressures delivered by nasal high flow oxygen during all phases of the respiratory cycle. Respir Care. 2013, 58:1621-24. 10.4187/respcare.02358

5. Frat JP, Coudroy R, Marjanovic N, Thille AW: High-flow nasal oxygen therapy and noninvasive ventilation in the management of acute hypoxemic respiratory failure. Ann Transl Med. 2017, 5:297.

10.21037/atm.2017.06.52 
6. Riera J, Pérez P, Cortés J, Roca O, Masclans JR, Rello J: Effect of high-flow nasal cannula and body position on end-expiratory lung volume: a cohort study using electrical impedance tomography. Respir Med. 2013, 58:589-96. 10.4187/respcare.02086

7. Roca O, Pérez-Terán P, Masclans JR, Pérez L, Galve E, Evangelista A, Rello J: Patients with New York Heart Association class III heart failure may benefit with high flow nasal cannula supportive therapy: high flow nasal cannula in heart failure. J Crit Care. 2013, 28:741-46. 10.1016/j.jcrc.2013.02.007

8. Möller W, Celik G, Feng S, et al.: Nasal high flow clears anatomical dead space in upper airway models . J Appl Physiol (1985). 2015, 118:1525-32. 10.1152/japplphysiol.00934.2014

9. Osadnik CR, Tee VS, Carson-Chahhoud KV, Picot J, Wedzicha JA, Smith BJ: Non-invasive ventilation for people with respiratory failure due to exacerbation of chronic obstructive pulmonary disease. Cochrane Database Syst Rev. 2017, 7:CD004104. 10.1002/14651858.CD004104.pub4

10. Frat JP, Thille AW, Mercat A, et al.: High-flow oxygen through nasal cannula in acute hypoxemic respiratory failure. N Engl J Med. 2015, 372:2185-96. 10.1056/NEJMoa1503326

11. Ni YN, Luo J, Yu H, et al.: Can high-flow nasal cannula reduce the rate of endotracheal intubation in adult patients with acute respiratory failure compared with conventional oxygen therapy and noninvasive positive pressure ventilation? A systematic review and meta-analysis151. Chest. 2017, 151:764-75. 10.1016/j.chest.2017.01.004

12. Azoulay E, Lemiale V, Mokart D, et al.: Effect of high-flow nasal oxygen vs standard oxygen on 28-day mortality in immunocompromised patients with acute respiratory failure: the HIGH randomized clinical trial. JAMA. 2018, (Epub ahead of print):E1-E9. 10.1001/jama.2018.14282

13. Hernández G, Vaquero C, González P, et al.: Effect of postextubation high flow nasal cannula use conventional oxygen therapy on reintubation in low-risk patients a randomized clinical trial. JAMA. 2016, 315:1354-61. 10.1001/jama.2016.2711

14. Ricard JD: Hazards of intubation in the ICU: role of nasal high flow oxygen therapy for preoxygenation and apneic oxygenation to prevent desaturation. Minerva Anestesiol. 2016, 82:1098-106.

15. Miguel-Montanes R, Hajage D, Messika J, et al.: Use of high-flow nasal cannula oxygen therapy to prevent desaturation during tracheal intubation of intensive care patients with mild-to-moderate hypoxemia. Crit Care Med. 2015, 43:574-83. 10.1097/CCM.0000000000000743

16. Ni YN, Luo J, Yu H, et al.: The effect of high-flow nasal cannula in reducing the mortality and the rate of endotracheal intubation when used before mechanical ventilation compared with conventional oxygen therapy and noninvasive positive pressure ventilation. A systematic review and meta-analysis. Am J Emerg Med. 2018, 36:226-33. 10.1016/j.ajem.2017.07.083

17. Peters SG, Holets SR, Gay PC: High-flow nasal cannula therapy in do-not-intubate patients with hypoxemic respiratory distress. Respir Care. 2013, 58:597-600.

18. Makdee O, Monsomboon A, Surabenjawong U, et al.: High-flow nasal cannula versus conventional oxygen therapy in emergency department patients with cardiogenic pulmonary edema: a randomized controlled trial. Ann Emerg Med. 2017, 70:465-472.e2. 10.1016/j.annemergmed.2017.03.028

19. Franklin D, Babl FE, Schlapbach LJ, et al.: A randomized trial of high-flow oxygen therapy in infants with bronchiolitis. N Engl J Med. 2018, 378:1121-31. 10.1056/NEJMoa1714855

20. Kepreotes E, Whitehead B, Attia J, et al.: High-flow warm humidified oxygen versus standard low-flow nasal cannula oxygen for moderate bronchiolitis (HFWHO RCT): an open, phase 4, randomised controlled trial. Lancet. 2017, 389:P930-39. 10.1016/S0140-6736(17)30061-2

21. Schibler A, Pham TM, Dunster KR, Foster K, Barlow A, Gibbons K, Hough JL: Reduced intubation rates for infants after introduction of high-flow nasal prong oxygen delivery. Intensive Care Med. 2011, 37:847-52. 10.1007/s00134-011-2177-5

22. Wing R, James C, Maranda LS, Armsby CC: Use of high-flow nasal cannula support in the emergency department reduces the need for intubation in pediatric acute respiratory insufficiency. Pediatr Emerg Care. 2012, 28:1117-23. 10.1097/PEC.0b013e31827122a9

23. Milési C, Boubal M, Jacquot A, Baleine J, Durand S, Odena MP, Cambonie G: High-flow nasal cannula: recommendations for daily practice in pediatrics. Ann Intensive Care. 2014, 4:29. 10.1186/s13613-014-00295

24. Schlapbach LJ, Schaefer J, Brady AM, Mayfield S, Schibler A: High-flow nasal cannula (HFNC) support in interhospital transport of critically ill children. Intensive Care Med. 2014, 40:592-99. 10.1007/s00134-0143226-7 